Trauma Berufskrankh 2008 10 [Suppl 3]:308-310

DOI 10.1007/s10039-008-1395-8

Online publiziert: 17. April 2008

๑) Springer Medizin Verlag 2008

\author{
S. Studier-Fischer \\ Klinik für Unfallchirurgie und Orthopädie, BG-Unfallklinik Ludwigshafen, \\ Unfallchirurgische Klinik an der Universität Heidelberg, Ludwigshafen
}

\title{
Zusammenhangstrennung der Rotatorenmanschette
}

\section{Diagnostik}

\section{Vorbemerkung}

Die Rotatorenmanschette ist eine Sehnenplatte der Mm. subscapularis, supraspinatus, infraspinatus und teres minor. Funktionell wird die Sehne des Caput longum M. bicipitis brachii hinzugezählt.

Über die Rotatorenmanschette setzt die tiefe Schultermuskulatur am Oberarmkopf an und zentriert und stabilisiert diesen in der Schulterpfanne. Das Sehnengewebe der Rotatorenmanschette besteht aus mehreren Schichten als Ausdruck der unterschiedlichen mechanischen Belastung. Die Supraspinatussehne ist in ihrem oberflächlichen Bereich eine Zugsehne und in der Tiefe eine Gleitsehne.

Defekte der Rotatorenmanschette gehören zu den häufigsten krankhaften Befunden des Schultergelenks. Meist sind sie degenerativer Art [3]. Bevor jedoch Begehrlichkeiten geweckt werden, sollte - auch wenn diese Begriffe im deutschen Sprachgebrauch ungewöhnlich klingen besser von einer Zusammenhangstrennung oder von einer Kontinuitätsunterbrechung gesprochen werden.

\section{Problematik}

Die gutachterliche Bewertung einer $\mathrm{Zu}$ sammenhangstrennung ist schwierig und häufig Grundlage und Anlass für rechtliche Auseinandersetzungen. Ziel muss es sein, in der Begutachtung bei gleichen medizinischen Sachverhalten einheitliche Beurteilungen abzugeben [1].

\section{Diagnose}

\section{Erste Informationen}

Die Eigenanamnese beinhaltet neben besonderen beruflichen Belastungen die sportliche Aktivität. Hierzu ist z. B. zu erfragen, ob die betroffene Person Arm belastende Sportarten ausführt oder ausführte; hierunter zählen z. B. Handball und Kampfsport - speziell Judo.

Befunde der Gegenseite und der angrenzenden Gelenke sind festzuhalten. Bereits im Durchgangsarztbericht (DArzt-Bericht) ist festzulegen, ob überhaupt ein Unfall im Sinne des Gesetzes vorliegt oder ob nicht ein willentlicher Vorgang beschrieben wird. Die Richtung der einwirkenden Kraft oder das Auftreffen des Körpers auf den Widerstand müssen klar und unmissverständlich dokumentiert werden. Von hohem Interesse ist der Hinweis auf eine stattgehabte Luxation. Der Schmerzverlauf ist wichtig; initial schmerzarme Zeiten von einigen Stunden oder Tagen sind genauso zu schriftlich zu fixieren wie ein sofortiges Schmerzmaximum.

Immer wieder wird vergessen, das Verhalten des Betroffenen nach dem Un- fall festzuhalten. Dabei ist zu erfassen, ob der Patient seine Arbeit fortgesetzte und wann er erstmals einen Arzt aufsuchte. Auch nicht D-ärztliche Konsultationen sind bedeutend. Dabei sind „innerbetriebliche“ Zwänge zu berücksichtigen, wie drohende Kündigungen oder hohe Auftragslage, bei welchen die Patienten erheblich unter Druck gesetzt werden können [2].

\section{Klinischer Erstbefund}

Im D-Arzt-Bericht muss aufgeführt werden, ob der Patient Schürfmarken oder Hämatome aufweist. Auch das Nichtvorliegen solcher Veränderungen ist von Bedeutung.

Bei der klinischen Untersuchung sind der passive Dehnungsschmerz und das Drop-Arm-Phänomen von immenser Bedeutung. Die Dokumentation des Bewegungsausmaßes nach der Neutral-o-Methode ist obligat.

Bei der Bewertung der Begleitverletzungen ist dem Akromioklavikulargelenk (AC-Gelenk) besonderes Augenmerk zu schenken. Auf neurologische Ausfälle z. B. am Plexus brachii oder am N. axillaris ist zu achten.
Tab. 1 Diagnostisches Stufenschema bei möglichem Rotatorenmanschettenschaden

\begin{tabular}{ll} 
Unfallzeitpunkt & Erstkonsultation \\
\cline { 2 - 2 } 2 Wochen nach dem Unfall & Dokumentation: Was liegt vor und was nicht? \\
\cline { 2 - 2 } & Zweitkonsultation, Befunde im Verlauf \\
\cline { 2 - 2 } & Verlaufsröntgen, erneute Sonographie \\
\cline { 2 - 2 } 4 Wochen nach dem Unfall & Veranlassung weitergehender Untersuchungen \\
\cline { 2 - 2 } & Definitive Festlegung über Schaden an der Rotatorenmanschette \\
\hline
\end{tabular}


Abb. 1 Röntgenaufnahme der Schulter mit Projektion des Humeruskopfs über dem Glenoid - Luxation damit nicht ausschließ bar: inakzeptable Qualität der Röntgenaufnahme

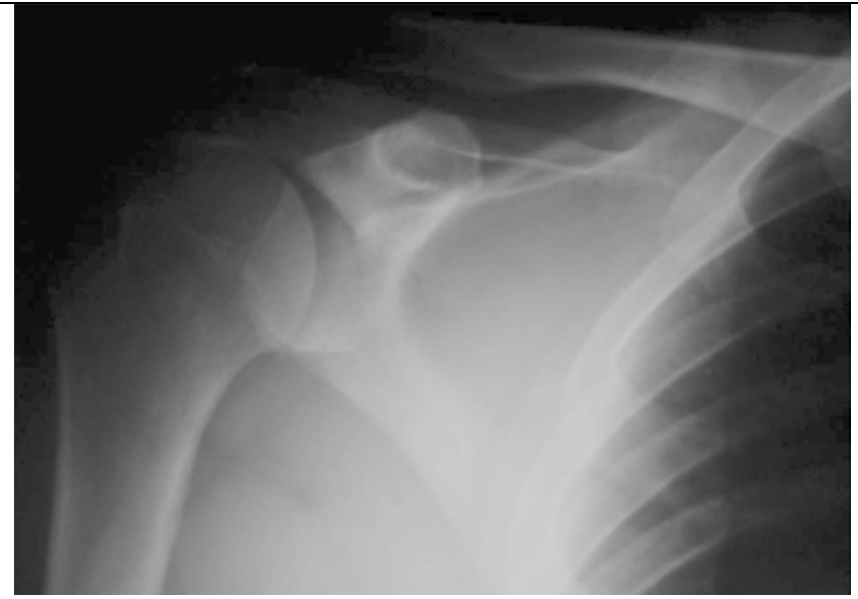

Abb. $2>$ Röntgenaufnahme der Schulter, eingestauchte Fraktur des Humeruskopfs

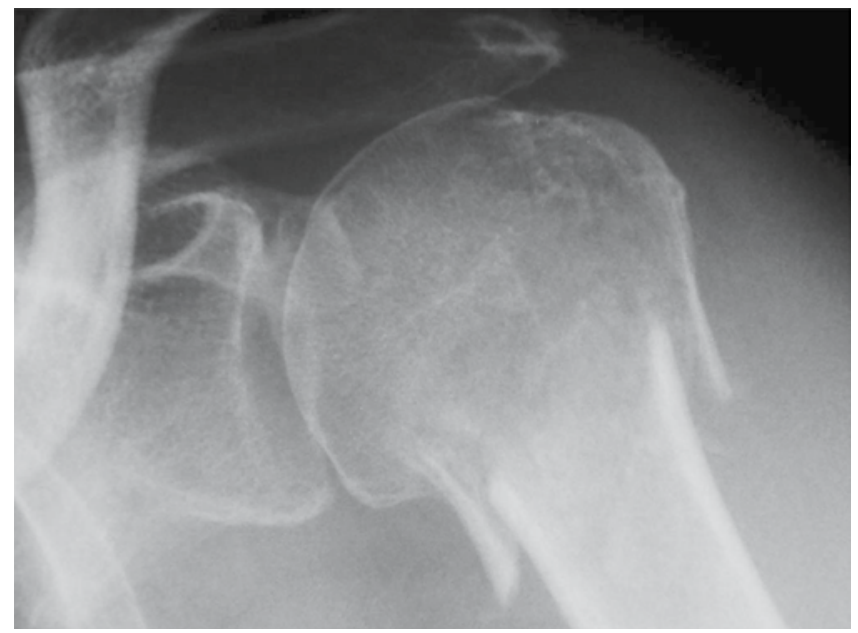

\section{Bildgebung}

Erste Röntgenaufnahme. Initial werden Röntgenaufnahmen in 2 Ebenen angefertigt, evtl. ist auch eine Outlet-ViewAufnahme erforderlich. Dabei ist auf eine exakte Einstellung des Gelenkspalts zu achten (• Abb. 1). Die Abbildungsqualität sollte die knöchernen Strukturen des Schultergelenks darstellen. Insbesondere müssen der subakromiale Raum und dessen Weite zu erkennen sein. Frakturen z. B. an Humeruskopf, Klavikula und Glenoid sind immer auszuschließen (• Abb. 2). Die Standartröntgenaufnahme gibt Hinweise auf eine vorliegende Omarthrose, Humeruskopfhochstand oder eine bereits vorliegende Cuff-Tear-Arthropathie als Zeichen eines fortgeschrittenen Rotatorenmanschettenschadens.

Sonographie. Wenngleich leicht durchzuführen, ist diese Untersuchung extrem untersucherabhängig. Sie ist sehr gut als „Kontaktaufnahme“ zum Patienten geeig- net. Aus persönlicher Erfahrung ist festzustellen, dass Patienten, welche sonographisch an der Schulter untersucht wurden, Vertrauen zum Untersucher aufbauen.

Mit hoher Sicherheit kann der Nachweis einer intakten Rotatorenmanschette erbracht werden. Das isolierte Vorliegen eines Ergusses ist nicht richtungsführend. Die Sonographie ist gleichzeitig eine dynamische Untersuchung, welche einen sehr guten Überblick über das Gleitverhalten des Gewebes und mögliche degenerative Veränderungen oder „Kalkherde“ bietet. Eine Aussage über das ACGelenk ist sofort möglich. Standardisiert muss die Gegenseite mituntersucht werden (• Abb. 3).

Magnetresonanztomographie. Mit oder ohne Kontrastmittel ist sie das sensibelste Verfahren zur Untersuchung der Rotatorenmanschette (• Abb. 4). Hiermit gelingen nichtinvasiv der Nachweis oder der Ausschluss von Rotatorenmanschettenschäden. Eine Retraktion der Sehnenrän-
Trauma Berufskrankh 2008 10

[Suppl 3]:308-310

DOI 10.1007/s10039-008-1395-8

๑) Springer Medizin Verlag 2008

\section{S. Studier-Fischer}

\section{Zusammenhangstrennung der Rotatorenmanschette. Diagnostik}

\section{Zusammenfassung}

Hat der Patient nach einem angeschuldigten Ereignis persistierende Beschwerden an der Schulter, ist seine Eigenanamnese zu erfragen. Befunde der Gegenseite, der Halswirbelsäule (HWS) und der angrenzenden Gelenke sind zu dokumentieren. Der Unfallhergang ist für die Begutachtung und die Bewertung elementar. Es ist zwingend festzuhalten, ob überhaupt ein Unfall vorliegt oder ob nicht eine willentliche Bewegung erfolgte. Die Richtung der einwirkenden Kraft und Hinweise auf stattgehabte Luxationen sind in jedem Fall bereits im ersten, dem so genannten Durchgangsarztbericht aufzuführen. Eine entsprechende Diagnostik ist ökonomisch einzusetzen und, falls erforderlich, zu wiederholen. 4 Wochen nach dem „Unfall" sollten die Diagnose feststehen und nach Bedarf eine entsprechende Therapie eingeleitet werden können.

\section{Schlüsselwörter}

Rotatorenmanschette $\cdot$ Ruptur . Begutachtung · Unfallabklärung · Durchgangsarztbericht

\section{Rotator cuff tear. Diagnosis}

\section{Abstract}

If a patient has continuous shoulder problems after an injury, the past medical history should be surveyed. Findings at other sites, the neck, and adjacent joints should be documented. Elementary for the appraisal is the mechanism of the injury itself. It is mandatory to know whether there is an injury at all or rather a voluntary motion. The direction of force and the findings of a former luxation are to be reported in the primary physician's report. Additional tests should be initiated economically and repeated if necessary. Four weeks after the so-called accident, the diagnostics should be completed, and therapy can be started.

\section{Keywords}

Rotator cuff · Cuff tear · Appraisal · Accident . Physician report 


\section{Verletzungen und Verletzungsfolgen am Schultergelenk}

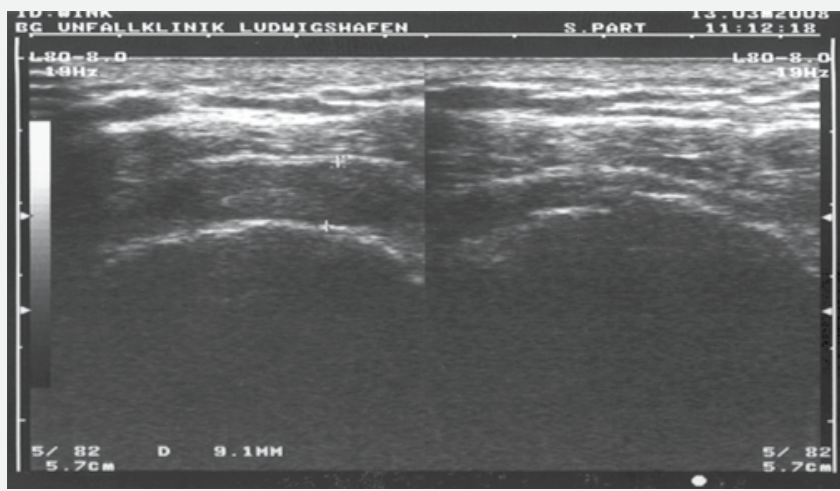

Abb. $3 \Delta$ Sonographische Aufnahme, nicht mehr nachweisbare Rotatorenmanschette der rechten Schulter
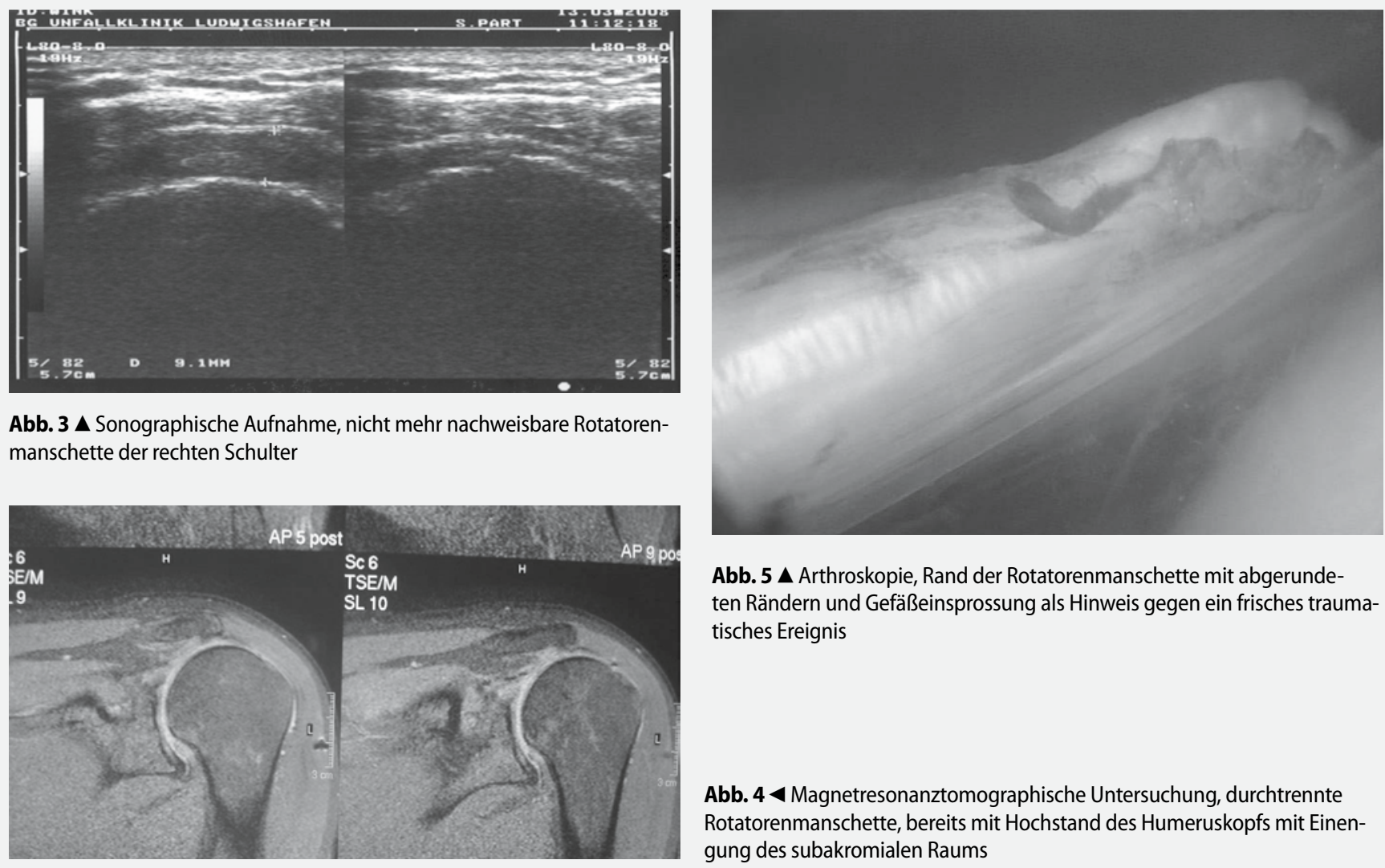

Abb. $5 \Delta$ Arthroskopie, Rand der Rotatorenmanschette mit abgerundeten Rändern und Gefäßeinsprossung als Hinweis gegen ein frisches traumatisches Ereignis

Abb. $4<$ Magnetresonanztomographische Untersuchung, durchtrennte Rotatorenmanschette, bereits mit Hochstand des Humeruskopfs mit Einengung des subakromialen Raums

der oder die fettige Degeneration der Sehne lassen sich sehr leicht erkennen. Auch Veränderungen am AC-Gelenk stellen sich sehr exakt dar. Ein Bone Bruise im Humeruskopf, eine Bankart-Läsion oder das Vorliegen einer Hill-Sachs-Delle sprechen für ein Unfallgeschehen.

Arthroskopie. Am Schluss der diagnostischen Kette, jedoch immer mit einer therapeutischen Intention, steht die Arthroskopie der Schulter. Dabei sind die Menge, die Farbe und die Konsistenz des Ergusses zu beschreiben. Der Defekt, falls vorliegend, wird ohne Wertung beschrieben (- Abb.5). Hierzu sind die Abrundung der Ränder, die Lage, die Richtung und Einblutungen anzugeben. Die Ausdehnung der Dehiszenz und die Adaptierbarkeit der Ränder geben dem Gutachter später Aufschluss über das Alter der Läsion. Die Beschreibung der Lage und des Zustands der langen Bizepssehne sowie der Begleitbefunde wie BankartSchaden, Hill-Sachs-Delle, SLAP-Läsion (SLAP: „superior labrum anterior posterior") usw. ist obligat.

\section{Histologie}

Eine repräsentative Entnahme ist zu fordern. Der Beauftragung der Histologie hat eine dezidierte Fragestellung zu folgen. Eine Entnahme später als 3 Monate nach einem fraglichen Ereignis lässt in der Regel keine zeitliche Zuordnung mehr zu. Das Labor sollte über eine entsprechende Erfahrung verfügen. Die Bedeutung der Histologie in der Zusammenhangsbegutachtung wird jedoch häufig überschätzt.

\section{Diagnostisches Stufenschema}

Wie üblich kommt der ersten Dokumentation im D-Arzt-Bericht und der Unfallmeldung eine enorme Bedeutung zu. Nicht alle Patienten bedürfen einer MRT. Eine Zweitkonsultation etwa 2 Wochen nach einem evtl. Unfall wird angeschlossen. Hier werden die ersten Untersuchungen ggf. wiederholt und Zweituntersuchungen veranlasst. Etwa 4 Wochen nach dem Unfall sollte die definitive Festlegung über den Schaden an der Rotatorenmanschette erfolgt sein. Im günstigen Fall kann dann unverzüglich mit der evtl. erforderlichen operativen Therapie begonnen werden ( $\mathbf{\bullet}$ Tab. 1).

\section{Korrespondenzadresse}

\section{Dr. S. Studier-Fischer}

Klinik für Unfallchirurgie und Orthopädie, BGUnfallklinik Ludwigshafen, Unfallchirurgische Klinik an der Universität Heidelberg, Ludwig-Guttmann-Straße 13,

67071 Ludwigshafen

stefan.studier-fischer@bgu-ludwigshafen.de

Interessenkonflikt. Der korrespondierende Autor gibt an, dass kein Interessenkonflikt besteht.

\section{Literatur}

1. Loew $M$, Habermeyer P, Wiedermann E et al. (2000) Empfehlungen zu Diagnostik und Begutachtung der traumatischen Rotatorenmanschettenläsion. Unfallchirurg 103: 417-426

2. Schönberger G, Mertens G, Valentin H (2003) Arbeitsunfall und Berufskrankheiten, 7. Aufl. Schmidt, Berlin

3. Weber M (2004) Empfehlungen zur Begutachtung von Schäden der Rotatorenmanschette. DGU Mitt Nachricht Suppl 2004: 27-33 